with withdrawal time $\leq 120$ minutes and location(s) of any polyps detected recorded. Primary outcome measures were mean number of polyps (MNP) and polyp detection rate (PDR). Multi-level mixed-effect negative binomial and logistic regression analyses, fitting endoscopist as a random effect, were used to examine associations between weekend procedure and these outcomes.

Results 92879 unique colonoscopies were performed by 2496 endoscopists. 19977 (21.5\%) were performed at weekends. Patients who had weekend procedures were more often female and less often seen for screening-related procedures $\left(\chi^{2}\right.$ tests: $\mathrm{P}<0.05)$; age differed little. Compared to weekday procedures, MNP was significantly lower for weekend procedures, after adjusting for patient age, sex and indication $(\mathrm{RR}=0.87,95 \%$ CI $0.84-0.90)$. A similar significant reduction was found for $\mathrm{PDR}$ at weekend $(\mathrm{OR}=0.86,95 \% \mathrm{CI} 0.82-0.90)$.

Abstract OTU-14 Table 1 Regression analyses: factors associated with MNP and PDR

\begin{tabular}{|c|c|c|c|c|}
\hline & \multicolumn{2}{|l|}{ MNP } & \multicolumn{2}{|l|}{ PDR } \\
\hline & Univariable & Multivariable & Univariable & Multivariable \\
\hline & $\mathrm{RR}(95 \% \mathrm{Cl})$ & RR $(95 \% \mathrm{Cl})$ & OR $(95 \% \mathrm{Cl})$ & OR $(95 \% \mathrm{Cl})$ \\
\hline \multicolumn{5}{|l|}{ Patient level } \\
\hline \multicolumn{5}{|l|}{ Weekend } \\
\hline \multicolumn{5}{|l|}{ procedure } \\
\hline No & 1.00 & 1.00 & 1.00 & 1.00 \\
\hline \multirow[t]{2}{*}{ Yes } & $0.81(0.78-$ & $0.87(0.84-$ & $0.81(0.78-$ & $0.86(0.82-$ \\
\hline & $0.84)^{a}$ & $0.90)^{a}$ & $0.85)^{a}$ & $0.90)^{a}$ \\
\hline \multicolumn{5}{|l|}{ Age, years } \\
\hline $18-39$ & - & 1.00 & - & 1.00 \\
\hline \multirow[t]{2}{*}{$40-49$} & - & 1.43 (1.35- & - & $1.62(1.50-$ \\
\hline & & $1.51)^{a}$ & & $1.74)^{a}$ \\
\hline \multirow[t]{2}{*}{$50-59$} & - & 2.16 & - & $2.67(2.50-$ \\
\hline & & $2.28)^{a}$ & & $2.85)^{a}$ \\
\hline \multirow[t]{2}{*}{$60-69$} & - & 2.66 & - & 3.51 (3.29- \\
\hline & & $2.80)^{a}$ & & $3.74)^{a}$ \\
\hline \multirow[t]{2}{*}{$\geq 70$} & - & 3.00 & - & 4.03 \\
\hline & & $3.15)^{a}$ & & $4.29)^{a}$ \\
\hline \multicolumn{5}{|l|}{ Sex } \\
\hline Women & - & 1.00 & - & 1.00 \\
\hline \multirow[t]{2}{*}{ Men } & - & 1.48 (1.45- & - & 1.60 \\
\hline & & $1.51)^{a}$ & & $1.65)^{\mathrm{a}}$ \\
\hline \multicolumn{5}{|l|}{ Indication } \\
\hline Other & - & 1.00 & - & 1.00 \\
\hline Lower GI & - & $0.79(0.76-$ & - & 0.77 (0.74- \\
\hline symptoms & & $0.81)^{a}$ & & $0.80)^{a}$ \\
\hline Previous & - & 1.27 (1.19- & - & 1.31 (1.19- \\
\hline abnormal & & $1.35)^{a}$ & & $1.43)^{a}$ \\
\hline \multicolumn{5}{|l|}{ investigation } \\
\hline \multirow[t]{2}{*}{ Previous polyp(s) } & - & 1.93 & - & $2.86(2.72-$ \\
\hline & & $2.00)^{a}$ & & $3.02)^{a}$ \\
\hline \multirow[t]{2}{*}{ IBD } & - & $0.53(0.49-$ & - & $0.44(0.40-$ \\
\hline & & $0.57)^{a}$ & & $0.49)^{a}$ \\
\hline \multirow[t]{2}{*}{ Screening } & - & 1.76 (1.68- & - & $2.53(2.37-$ \\
\hline & & $1.84)^{a}$ & & $2.71)^{a}$ \\
\hline Endoscopist & $0.51(0.02)$ & $0.27(0.01)$ & $0.55(0.02)$ & $0.39(0.02)$ \\
\hline \multicolumn{5}{|l|}{ level, variance } \\
\hline (SE) & & & & \\
\hline
\end{tabular}

This study, part of the NED-APRIQOT project, was funded by the Health Foundation.

Conclusions Colonoscopy polyp detection is significantly lower at weekends, after adjusting for case-mix. Research is needed to better understand the reasons for this unwarranted variation.

IBD

\section{OFR-8 INFLIXIMAB IS ASSOCIATED WITH ATTENUATED IMMUNOGENICITY TO BNT162B2 AND CHADOX1 NCOV-19 SARS-COV-2 VACCINES}

${ }^{1,2}$ Nicholas Kennedy, ${ }^{1,2}$ Simeng Lin*, ${ }^{1,2}$ James Goodhand, ${ }^{3,4}$ Nicholas Powell, ${ }^{1,2}$ Tariq Ahmad, CLARITY IBD study group. 'Department of Gastroenterology, Royal Devon and Exeter NHS Foundation Trust, Exeter, UK; ${ }^{2}$ Exeter Inflammatory Bowel Disease and Pharmacogenetics Research Group, University of Exeter, Exeter, UK; ${ }^{3}$ Department of Gastroenterology, Imperial College Healthcare NHS Trust, London, UK; ${ }^{4}$ Department of Metabolism, Digestion and Reproduction, Imperial College London, London, UK

\subsection{6/gutjnl-2021-BSG.5}

Introduction Pressure on critical care services because of further COVID-19 surges have forced governments to prioritise primary vaccination to vulnerable groups. To extend limited vaccine supplies, many countries including the United Kingdom have also delayed second vaccine doses, trading maximal effectiveness for a lower level of protective immunity across a greater proportion of the most at-risk population. We investigated whether patients with inflammatory bowel disease (IBD) treated with infliximab have attenuated serological responses to a single-dose of a SARSCoV-2 vaccine.

Methods Antibody responses and seroconversion rates in infliximab-treated patients $(n=865)$ were compared to a cohort treated with vedolizumab $(n=428)$, a gut-selective antiintegrin $\alpha 4 \beta 7$ monoclonal antibody. Our primary outcome was anti-SARS-CoV-2 spike (S) antibody concentrations 3-10 weeks after vaccination in patients without evidence of prior infection. Secondary outcomes were seroconversion rates defined by a cut-off of $15 \mathrm{U} / \mathrm{mL}$, and antibody responses following past infection or a second dose of the BNT162b2 vaccine.

Results Geometric mean [SD] anti-SARS-CoV-2 antibody concentrations were lower in patients treated with infliximab than vedolizumab, following BNT162b2 (6.0 U/mL [5.9] vs $28.8 \mathrm{U} /$ $\mathrm{mL}$ [5.4] $\mathrm{P}<0.0001)$ and ChAdOx1 nCoV-19 (4.7 U/mL [4.9]) vs $13.8 \mathrm{U} / \mathrm{mL}$ [5.9] $\mathrm{P}<0.0001)$ vaccines. In our multivariable models, antibody concentrations were lower in infliximabcompared to vedolizumab-treated patients who received the BNT162b2 (fold change [FC] 0.29 [95\% CI $0.21,0.40]$, $\mathrm{p}<0.0001$ ) and ChAdOx1 nCoV-19 (FC 0.39 [95\% CI 0.30 , 0.51 ], $\mathrm{p}<0.0001)$ vaccines. In both models, age $\geq 60$ years, immunomodulator use, Crohn's disease, and smoking were associated with lower, whilst non-white ethnicity was associated with higher, anti-SARS-CoV-2 antibody concentrations. Seroconversion rates after a single-dose of either vaccine were higher in patients with prior SARS-CoV-2 infection and after two doses of BNT162b2 vaccine (Figure 1).

Conclusions Infliximab is associated with attenuated immunogenicity to a single-dose of the BNT162b2 and ChAdOx1 nCoV-19 SARS-CoV-2 vaccines. Vaccination after SARS-CoV-2 infection, or a second dose of vaccine, led to seroconversion in most patients. Delayed second dosing should be avoided in patients treated with infliximab. 


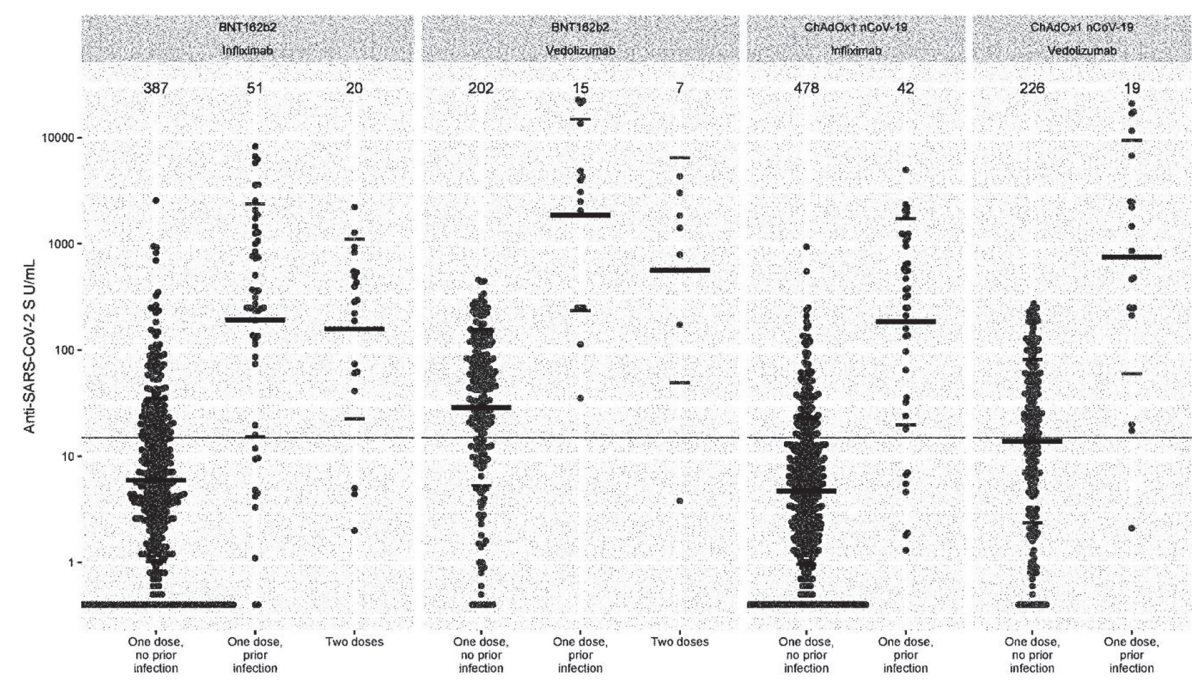

Abstract OFR-8 Figure 1

\section{OFR-9 AN RCT OF AUTOLOGOUS STEM-CELL TRANSPLANTATION IN TREATMENT REFRACTORY CROHN'S DISEASE (LOW-INTENSITY THERAPY EVALUATION): ASTICLITE}

\begin{abstract}
${ }^{1}$ James Lindsay*, ${ }^{2}$ Shahida Din, ${ }^{3}$ Chris Hawkey, ${ }^{4}$ Danny Hind, ${ }^{5}$ Peter Irving, ${ }^{4}$ Alan Lobo, ${ }^{3}$ Yash Mahida, ${ }^{3}$ Gordon Moran, ${ }^{6}$ Miles Parkes, ${ }^{7}$ Jack Satsangi, ${ }^{8}$ Sree Subramanian, ${ }^{4}$ Lizzie Swaby, ${ }^{7}$ Simon Travis, ${ }^{4}$ John Snowden. ${ }^{1}$ Barts Health NHS Trust, London, UK; ${ }^{2}$ Western General Hospital, Edinburgh, UK; ${ }^{3}$ University of Nottingham, Nottingham, UK; ${ }^{4}$ University of Sheffield, Sheffield, UK; ${ }^{5}$ Guy's and St Thomas' Hospital, London, UK; ${ }^{6}$ Cambridge University Hospitals, Cambridge, UK; ${ }^{7}$ Oxford University Hospitals, Oxford, UK; ${ }^{8}$ Royal Liverpool University Hospital, Liverpool, UK
\end{abstract}

\subsection{6/gutjnl-2021-BSG.6}

Introduction Reports of benefit from HSCT were tempered by the ASTIC trial which failed its ambitious primary endpoint and reported high toxicity. Subsequent reports suggest that HSCT achieves complete mucosal healing in 50\%, and that toxicity relates to the cyclophosphamide dose.

Design A UK multi-site RCT comparing low intensity HSCT with standard of care (SOC) in patients with active CD at endoscopy (SESCD ulcer score $\geq 2$ ) refractory to 2 biologic classes. Planned recruitment was 99 patients randomised 2:1 to HSCT versus SOC. The primary endpoint was centrally read endoscopic remission (SESCD ulcer subscore of 0 ) without requirement for surgery or death at week 48 .

Results The trial was halted due to unexpected SAE after 23 patients (13 HSCT, 10 SOC) had been randomised. The coronavirus pandemic prevented some outcome assessments. Patients had advanced disease: mean (SD) CD duration 13.9 (7) years; CDAI at baseline 337.5 (182.4) with 20 (91\%) having undergone surgery and $9(43 \%)$ having a stoma. All patients contributed to the safety analysis. The primary outcome using central reading was available for 7/10 HSCT and 6/9 SOC patients. Absence of endoscopic ulceration without surgery or death was reported in $3 / 7$ (43\%) HSCT patients compared to $0 / 9(0 \%)$ SOC patients. Centrally read SESCD (mean (SD)) was 11.8 (8.7) and 10.1 (5.7) at baseline compared to $2.8(2.9)$ and $18.7(9.1)$ at week 48 in the HSCT and SOC groups respectively. Clinical remission (CDAI <150) occurred in $57 \%$ and $0 \%$ of patients in the HSCT and SOC groups at week 48. SAE were more frequent after HSCT (39 in $13(100 \%)$ patients) than SOC (15 in 4 (40\%) patients).
Importantly, 10 SUSARs were reported in 6 HSCT patients including 3 cases of delayed renal failure due to thrombotic microangiopathy (TMA). Two patients in the HSCT arm died. Conclusion HSCT using a low intensity regimen results in meaningful reduction in endoscopic disease activity with some patients experiencing resolution of ulceration. However, the incidence of serious adverse events makes the regimen used in this trial unsuitable for future clinical use.

This project $(15 / 178 / 09)$ is funded by the Efficacy and Mechanism Evaluation (EME) Programme, an MRC and NIHR partnership. The views expressed in this publication are those of the author(s) and not necessarily those of the MRC, NIHR or the Department of Health and Social Care.

\section{OFR-10 ADMISSION MODEL FOR INTENSIFICATION OF THERAPY IN ACUTE SEVERE COLITIS (ADMIT-ASC)}

${ }^{1}$ Alex Adams*, ${ }^{1}$ Vipin Gupta, ${ }^{2}$ Waled Mohsen, ${ }^{1}$ Thomas Chapman, ${ }^{2}$ Deloshaan Subhaharan, ${ }^{2}$ Pradeep Ramaswamy, ${ }^{1}$ Oxford IBD Cohort Investigators, ${ }^{3}$ Vineet Ahuja, ${ }^{1}$ Simon Travis, ${ }^{1}$ Jack Satsangi. ${ }^{1}$ Translational Gastroenterology Unit, University of Oxford, Oxford, UK; ${ }^{2}$ Digestive Diseases Unit, Gold Coast University Hospital, Australia; ${ }^{3}$ Department of Gastroenterology, All India Institute of Medical Sciences, New Delhi, India

\subsection{6/gutjinl-2021-BSG.7}

Introduction Acute severe colitis (ASC) is an important cause of morbidity and mortality in UC, requiring hospitalisation and often colectomy. Accepted management is protocolised response assessment at Day 3 of IV steroid treatment. If steroid non-responders could be identified at all earlier stage, intensification may be possible prior to Day 3. It is also unclear whether ASC outcomes have chaned over the past 25 years and we aimed to examine this.

Methods We examined ASC cohorts across 3 continents to produce an accurate predictor of steroid response. All patients received protocolised treatment including first-line IV corticosteroids, and endoscopic (UCEIS) scoring. Factors associated with requirement for rescue therapy, colectomy during admission, and the following year, and a composite measure of steroid response were identified by logistic regression in 131 adult ASC admissions in Oxford, UK, between 2015-9. 